\title{
Alternating Segmentation and Simulation for Contrast Adaptive Tissue Classification
}

\author{
Dzung L. Pham ${ }^{\mathrm{a}}$ and Snehashis Roy ${ }^{\mathrm{a}}$ \\ ${ }^{a}$ Center for Neuroscience and Regenerative Medicine, Bethesda, MD, USA
}

\begin{abstract}
A key feature of magnetic resonance (MR) imaging is its ability to manipulate how the intrinsic tissue parameters of the anatomy ultimately contribute to the contrast properties of the final, acquired image. This flexibility, however, can lead to substantial challenges for segmentation algorithms, particularly supervised methods. These methods require atlases or training data, which are composed of MR image and labeled image pairs. In most cases, the training data are obtained with a fixed acquisition protocol, leading to suboptimal performance when an input data set that requires segmentation has differing contrast properties. This drawback is increasingly significant with the recent movement towards multi-center research studies involving multiple scanners and acquisition protocols. In this work, we propose a new framework for supervised segmentation approaches that is robust to contrast differences between the training MR image and the input image. Our approach uses a generative simulation model within the segmentation process to compensate for the contrast differences. We allow the contrast of the MR image in the training data to vary by simulating a new contrast from the corresponding label image. The model parameters are optimized by a cost function measuring the consistency between the input MR image and its simulation based on a current estimate of the segmentation labels. We provide a proof of concept of this approach by combining a supervised classifier with a simple simulation model, and apply the resulting algorithm to synthetic images and actual MR images.
\end{abstract}

Keywords: magnetic resonance imaging, machine learning, contrast adaptation, segmentation, simulation

\section{DESCRIPTION OF PURPOSE}

Magnetic resonance (MR) imaging plays a critical role in diagnosis, monitoring disease progression, and response to therapies. Automated segmentation algorithms for quantifiying brain structure in MR images have shown increasing promise for routine application in both research studies and clinical evaluations of neurodegenerative diseases. The best performing algorithms currently rely on training data or atlases to serve as exemplars of how MR images should be segmented. Unfortunately, such approaches perform suboptimally and inconsistently when faced with imaging data that possess contrast properties that differ from the available exemplars. This weakness serves as a substantial disadvantage in longitudinal studies that are subject to scanner upgrades and continually evolving imaging protocols. Furthermore, in the age of "big data," there is a huge demand to pool data across multiple studies and sites for increasing statistical power. However, in a recent study scanning a patient at 7 different sites across North America, even with careful matching of the MRI pulse sequence, variations across sites were significant [1].

An example of the sensitivity of segmentation algorithms to MRI contrast is shown in Fig. 1, where two T1-w images are acquired from the same subject. Despite the fact that the underlying anatomy should be the same, when three different segmentation algorithms were applied (FreeSurfer, ${ }^{2}$ S3DL, ${ }^{3}$ and Label Fusion ${ }^{4}$ ), each yielded substantially different results depending on the contrast. In all cases, the second T1-weighted image yielded a thicker cerebral cortex with smaller sulcal gaps. Note also that the different algorithms exhibit different sensitivities to the contrast differences, with the S3DL approach showing the most dramatic differences. As a patch-based approach, it is more dependent on the intensity variations of its training data, leading to greater sensitivity to contrast differences.

Multiple approaches have been previously proposed to perform contrast or intensity normalization. The most common approach to compensating for site differences is to employ a site covariate within the statistical modeling $[5,6]$. However, this has not been well validated, and there is limited evidence to suggest that imaging 


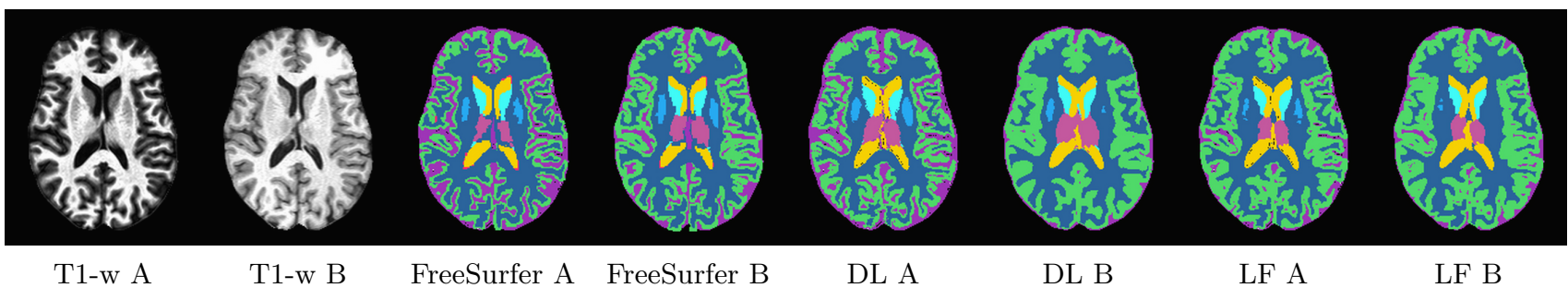
T1-w A
T1-w B
FreeSurfer A FreeSurfer B
DL A
DL B
LF A
LF B

Figure 1. Segmentation results given two different T1-w scans. DL stands for dictionary learning ${ }^{3}$ and LF stands for Label Fusion. ${ }^{4}$

variations are well captured by such an approach. Intensity normalization techniques have also been proposed [7, 8] that attempt to align the histograms of images using linear or piecewise linear transformations. Because the transformations affect the global histogram, local contrast differences that are region specific are not addressed by this approach. Furthermore, there is an inherent assumption that the global histogram is similar in the two images being matched. In the case where one image possesses pathology and the other does not, this assumption is violated.

Image synthesis is a class of image processing techniques where images from a subject are used in combination with training data to create a new image with desirable intensity properties [9-11]. A matching process is performed between the input images and the training data images with the same contrast, and an output image with the desired contrast properties is computed. The advantage of image synthesis for contrast normalization over histogram matching approaches is that the transformation can be based on local properties of the images, and is non-parametric. Because of this greater flexibility, synthesis has been shown to outperform global histogram normalization methods for contrast normalization [10].

In this work, we propose a novel framework to directly integrate a generative MR simulation into the segmentation process. We use the MR simulation process to generate new images within the training data from the label images. As a proof of concept, we adopt a simple simulation model and show that by minimizing the error between the input image and its simulated version based on the current segmentation estimate, contrast differences between the training and input images can be effectively addressed. Preliminary results applying the approach to synthetic and real MR images demonstrate substantial improvement in obtaining a consistent segmentation, on the same level as when the training and input images have the same contrast. Our approach bares conceptual similarites to generative adversarial networks [12], which also employs both generative and discriminative processes, but the rationale and implementation are different here.

\section{METHODS}

We denote a segmentation $\mathcal{D}(\cdot)$ to be a mapping from the MR image to its anatomical labels, which represent the desired regions of interest. We consider the segmentation to be a supervised process that requires a training data set consisting of MR image and label image pairs. We denote the simulation $\mathcal{G}(\cdot ; \boldsymbol{\theta})$ to be a mapping from the labels to an MR image, parameterized by the vector $\boldsymbol{\theta}$. Our combined segmentation and simulation framework is illustrated in Fig. 2. Note that the training label images and subject images are fixed, while the training MR images and subject labels are updated with each iteration. In the first iteration, the segmentation is performed as usual

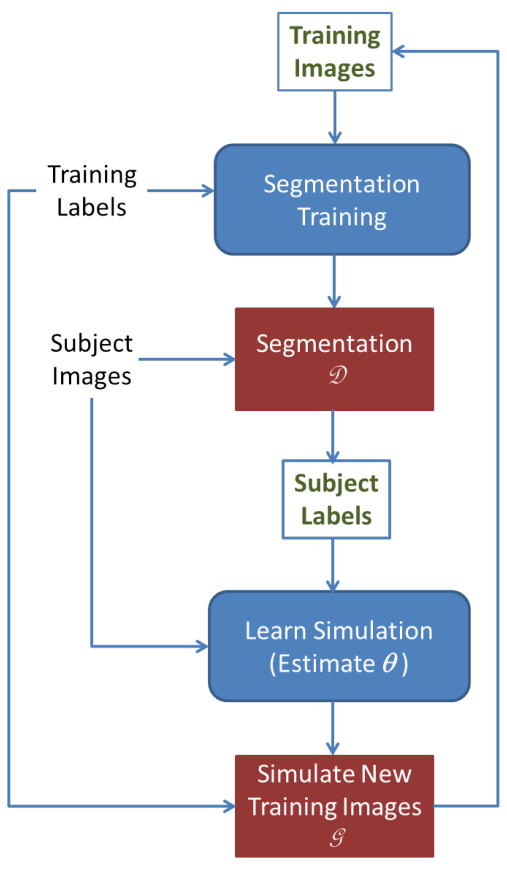

Figure 2. Block diagram of contrast adaptive framework with the available training data, yielding an initial segmentation estimate. This estimate is then used to compute the parameters within the simulation process by minimizing a consistency equation. Given the input MR image $X$ and segmentation estimate $L$, we estimate $\boldsymbol{\theta}$ according to

$$
\hat{\boldsymbol{\theta}}=\arg \min _{\theta}\|X-\mathcal{G}(L ; \boldsymbol{\theta})\|^{2}
$$


Using the estimated simulation parameters, we generate new MR images from the label images within the training data, which are subsequently used to segment the input image again. This process iterates until minimal changes occur within the segmentation.

Because the purpose of this work is to primarily serve as a proof of concept of this approach, we use a relatively simple classifier for $\mathcal{D}$ and a simple simulation model for $\mathcal{G}$. Although more sophisticated models, such as deep learning networks, can be used, our simplified choices allow the proposed framework to be more conveniently demonstrated. For $\mathcal{D}$, we use a Gaussan classifier for segmentation. A Gaussian classifer in this context models each tissue class as a Gausian distribution, given by,

$$
p_{j k}=\frac{1}{\sqrt{2 \pi \sigma^{2}}} \exp \frac{\left(x_{j}-\mu_{k}\right)^{2}}{2 \sigma_{k}^{2}}
$$

where $\mu_{k}$ and $\sigma_{k}^{2}$ are the mean and variance of the intensities for each tissue class $k$ and are estimated from the training data. The variable $j$ represents the pixel or voxel location. A standard segmentation approach would use the estimated values of $\mu_{k}$ and $\sigma_{k}^{2}$ from the training data to compute the probability that each voxel in the input image $X$ belongs to tissue class $k$ and stop at that point. A hard label image can be generated by assigning each voxel to the tissue class of highest probability.

Under our framework, we alternate the classification step with a simulation step. Given the input image, and an estimate of the segmentation probability functions, we seek to define the simulation process that maps the segmentation to the intensities. For the simulation model, we assume that each pixel is a linear combination of a tissue class centroid weighted by the probability or partial volume fraction. This is given by

$$
y_{j}=\sum_{k=1}^{K} p_{j k} c_{k}
$$

where $y_{j}$ is the pixel or voxel intensity at location $j, p_{j k}$ is the probability or partial volume fraction that voxel $j$ belongs to label or class $k$, and $c_{k}$ is the intensity of label $k$. For simplicity, noise effects are ignored in the model.

Under this model, $\boldsymbol{\theta}=\left[c_{1}, \ldots, c_{k}\right]^{T}$ and it is straightforward to show that Eq. 1 is solved by

$$
\boldsymbol{\theta}=\left(P^{T} P\right)^{-1} P^{T} \boldsymbol{x}
$$

where $P$ is the matrix of probabilities $p_{j k}$, and $\boldsymbol{x}$ is a stacking of the input MR image pixels $x_{j}$. For estimating $\boldsymbol{\theta}$, the probabilities are taken from the Gaussian classifier segmentation result. We note that $c_{k}$ is computed from the input image, while $\mu_{k}$ is computed from the training data. For generating new MR images in the training data, we apply Eq. 3 assuming that the probabilities are provided within the label images of the training data, or, if unavailable, derived from a blurring filter applied to the hard labels.

\subsection{New or breakthrough work to be presented}

The inconsistent performance of supervised segmentation algorithms when applied to images with different contrast properties is a critical issue in medical image analysis. This problem manifests itself in two common ways. First, if the contrast of an input image is different from that of the training data, performance is compromised, generally leading to reduced segmentation accuracy and rendering tools for detecting subtle differences in anatomical structure unusable. Second, when attempting to analyze data acquired with different protocols or from from different sites, standard approaches will lead to significant biases in the segmentation results that prohibit direct comparisons and pooling of data. This work helps restore the ability to find subtle relationships in pooled heterogeneous MR data sets by combining segmentation and simulation techniques. Although previous methods have been proposed for contrast matching, our approach benefits from being integrated into the segmentation process. This work has not been presented elsewhere. 


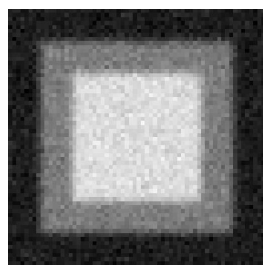

(a)

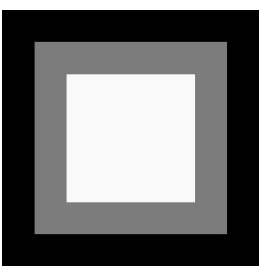

(b)

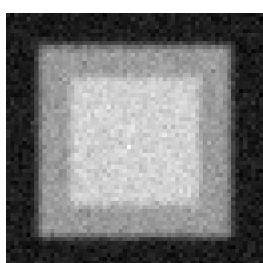

(c)

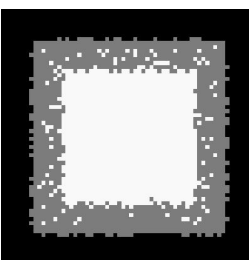

(d)

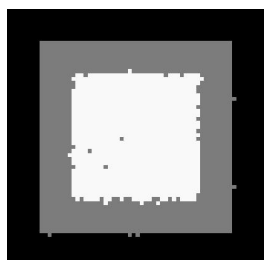

(e)

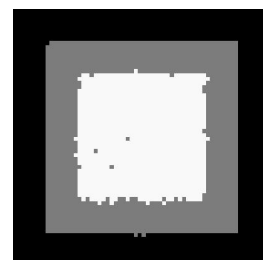

(f)

Figure 3. Segmentation results on a synthetic test image with 3 classes: (a) training image, (b) training labels, (c) test image, (d) standard segmentation, (e) proposed segmentation, (f) ideal segmentation.

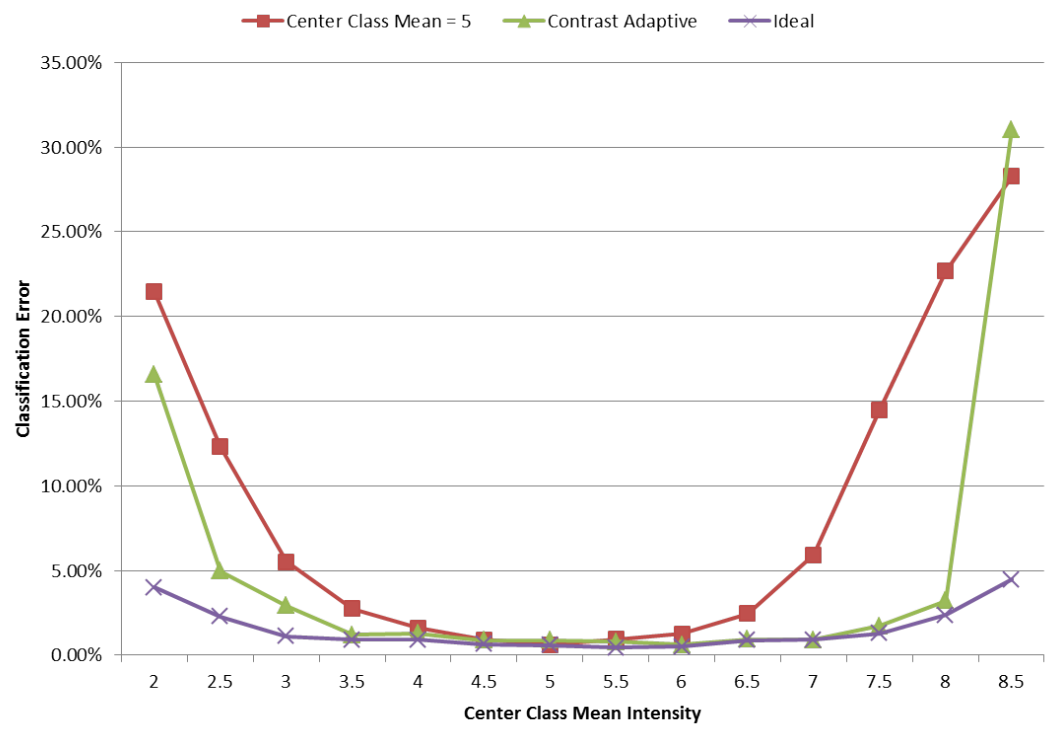

Figure 4. Classification errors as the mean of the center class from Fig. 3 varies from 2 to 8.5. The purple curve represents performance with ideal training data that varies according to the input. The green curve represents our proposed approach, and the red curve represents performance when the training data is fixed.

\section{RESULTS}

We first demonstrate our method using a synthetic test image shown in Fig. 3. The training image consisted of 3 classes with means 0,5, and 10, and included blurring and additive Gaussian noise with a standard deviation of 0.5. The test image had the center class mean altered, and in the case of Fig. 3(c), set to 7 rather than 5 . Applying a Gaussian classifer to the test image yielded poor results, as shown Fig. 3(d). Using our contrast adaptive framework, five iterations were required to convergence (defined as less $0.01 \%$ pixels changing labels). The resulting segmentation in Fig. 3(e) is very similar to the result one would obtain if the classifier were trained on data with the same contrast properties as the input, which we refer to as the "ideal" segmentation shown in Fig. 3(f).

Figure 4 shows how the classification error, defined as the ratio of misclassified pixels to total pixels, varies as the center class mean is changed from 2 to 8.5. The bottom curve represents the ideal segmentation error, where the training data has the same contrast as the test data. The top curve represent the scenario where the training data is fixed, with the center class mean equal to 5. The curve in between represents our proposed algorithm, which reduces the classification error as the class mean moves away from 5 . Note also that the performance of our algorithm is better at higher intensity values than lower ones. This is because at lower mean intensities, the contrast to noise ratio deteriorates more rapidly, with the magnitude of noise becoming almost equal to the magnitude of the signal difference between each class. Nevertheless, there does reach a point where the simulation step does not improve segmentation results and the error increases dramatically.

Fig. 5 shows the effect of applying our proposed approach to segment a T1-weighted MR image. Figs. 5(a) and 


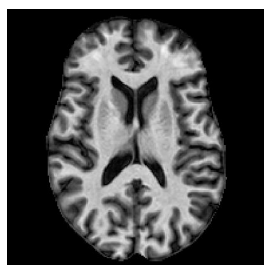

(a)

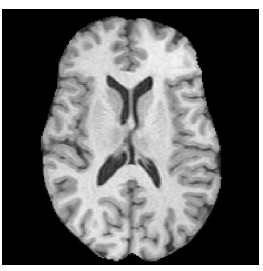

(b)

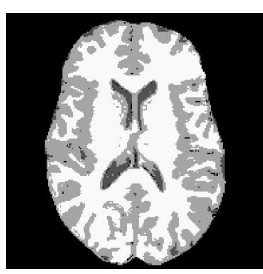

(c)

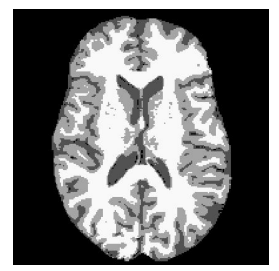

(d)

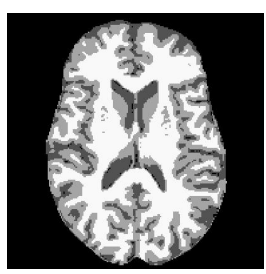

(e)

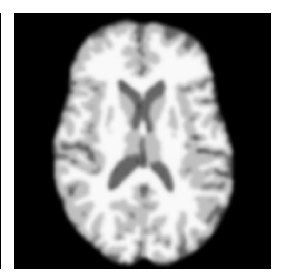

(f)

Figure 5. Segmentation results on MR images with different contrast properties: (a) MPRAGE training image, (b) SPGR test image, (c) standard segmentation of SPGR, (d) proposed segmentation of SPGR, (e) segmentation of MPRAGE image, (f) simulated SPGR image.

(b) show co-registered T1-weighted images acquired from the same subject. The former image is an MPRAGE acquisition, and the latter image is an SPGR, each leading to a rather distinct depiction of the brain anatomy. The images have been pre-processed for inhomogeneity correction and brain extraction. Using the MPRAGE as a training image for a three-class, supervised Gaussian classifier applied to the SPGR yields the result shown in Fig. 5(c). The lateral ventricles appear to be under segmented and sulcal cerebrospinal fluid (CSF) is almost entirely lacking. Using the proposed algorithm, one achieves a very similar segmentation result to what would be obtained if directly segmenting the MPRAGE image, as shown in Figs. 5(d) and (e). The Dice coefficents between these two segmentations are $0.81,0.81$, and 0.91 for CSF, gray matter, and white matter respectively. Comparing the MPRAGE segmentation to the standard SPGR segmentation, the Dice coefficents are 0.42, 0.69, and 0.88 . This amounts to an overall improvement of over $27 \%$ in segmentation consistency. The consistency in total brain (gray and white matter) estimated from the segmentations of the SPGR and the MPRAGE images without and with contrast adaptation is improved from $17.5 \%$ to $0.7 \%$, the latter value being within the range of typical segmentation error. ${ }^{13}$

Fig. 5(f) shows the final simulated MR image within the training data. The overall contrast of the training data has been transformed to be more similar to the SPGR, although there is ample room for improving the realism of the simulation. In particular, the simulation model employed here is quite rudimentary in comparison to other techniques that have been proposed, such as in [14].

\section{Conclusions}

Our preliminary data demonstrates improved robustness to contrast differences between training and test images when using our proposed framework. Better performance can likely be achieved by using more sophisticated techniques for both the segmentation and simulation. In future work, we will use deep learning networks for both the segmentation and simulation steps. A limitation of this work is that a whole brain segmentation in order to produce reasonable simulations. Its use with lesion detection techniques, for example, would require significant modifications.

\section{Acknowledgments}

This work was supported by the Department of Defense in the Center for Neuroscience and Regenerative Medicine, and by grant RG-1507-05243 from the National Multiple Sclerosis Society.

\section{REFERENCES}

[1] Shinohara, R., Calabresi, P., Davatzikos, C., J.Doshi, Henry, R., Kim, G., Linn, K., Nair, G., oh, J., Papinutto, N., Pham, D., Reich, D., Rooney, W., Roy, S., Sicotte, N., Stern, W., Tummala, S., Yousuf, F., Zhu, A., and Bakshi, R., "Volumetric analysis from a harmonized multisite brain MRI study of a single subject with multiple sclerosis," American Journal of Neuroradiology (in press).

[2] Fischl, B., "FreeSurfer," Neuroimage 62, 774-781 (Aug 2012).

[3] Roy, S., He, Q., Sweeney, E., Carass, A., Reich, D. S., Prince, J. L., and Pham, D., "Subject-Specific Sparse Dictionary Learning for Atlas-Based Brain MRI Segmentation," IEEE J Biomed Health Inform 19, 1598-1609 (Sep 2015). 
[4] Ledig, C., Heckemann, R. A., Hammers, A., Lopez, J. C., Newcombe, V. F., Makropoulos, A., Lotjonen, J., Menon, D. K., and Rueckert, D., "Robust whole-brain segmentation: application to traumatic brain injury," Med Image Anal 21, 40-58 (Apr 2015).

[5] Jones, B. C., Nair, G., Shea, C. D., Crainiceanu, C. M., Cortese, I. C., and Reich, D. S., "Quantification of multiple-sclerosis-related brain atrophy in two heterogeneous MRI datasets using mixed-effects modeling," Neuroimage Clin 3, 171-179 (2013).

[6] Chua, A. S., Egorova, S., Anderson, M. C., Polgar-Turcsanyi, M., Chitnis, T., Weiner, H. L., Guttmann, C. R., Bakshi, R., and Healy, B. C., "Handling changes in MRI acquisition parameters in modeling whole brain lesion volume and atrophy data in multiple sclerosis subjects: Comparison of linear mixed-effect models," Neuroimage Clin 8, 606-610 (2015).

[7] Nyul, L., Udupa, J., and Zhang, X., "New variants of a method of MRI scale standardization," IEEE transactions on medical imaging 19(2), 143-150 (2000).

[8] Shinohara, R. T., Sweeney, E. M., Goldsmith, J., Shiee, N., Mateen, F. J., Calabresi, P. A., Jarso, S., Pham, D. L., Reich, D. S., and Crainiceanu, C. M., "Statistical normalization techniques for magnetic resonance imaging," Neuroimage Clin 6, 9-19 (2014).

[9] Roy, S., Carass, A., and Prince, J., "Magnetic Resonance Image Example Based Contrast Synthesis," IEEE Trans Med Imaging 32, 2348 - 2363 (2013).

[10] Jog, A., Carass, A., Roy, S., Pham, D. L., and Prince, J. L., "MR image synthesis by contrast learning on neighborhood ensembles," Med Image Anal 24, 63-76 (May 2015).

[11] Jog, A., Carass, A., Roy, S., Pham, D. L., and Prince, J. L., "Random forest regression for magnetic resonance image synthesis," Med Image Anal 35, 475-488 (Jan 2017).

[12] Goodfellow, I., Pouget-Abadie, J., Mirza, M., Xu, B., Warde-Farley, D., Ozair, S., Courville, A., and Bengio, Y., "Generative adversarial nets," in [Advances in neural information processing systems], 2672-2680 (2014).

[13] Heinen, R., Bouvy, W. H., Mendrik, A. M., Viergever, M. A., Biessels, G. J., and de Bresser, J., "Robustness of Automated Methods for Brain Volume Measurements across Different MRI Field Strengths," PLoS ONE 11(10), e0165719 (2016).

[14] He, Q., Roy, S., Jog, A., and Pham, D., "An example-based brain MRI simulation framework," in [SPIE Medical Imaging], (Feb. 21-26 2015). 